pp. 195-203 in T.Placek and J. Butterfield (eds.): Non-locality and Modality,

NATO Science Series, Kluwer Academic Publishers, Dordrecht, 2002.

\title{
A Kochen-Specker Theorem for Unsharp Spin 1 Observables
}

\author{
Thomas Breuer \\ Department of Computer Science, FH Vorarlberg \\ A-6850 Dornbirn, Austria \\ thomas.breuer@fh-vorarlberg.ac.at
}

\begin{abstract}
Unsharp spin observables are shown to arise from the fact that a residual uncertainty about the actual alignment of the measurement device remains. If the uncertainty is below a certain level, and if the distribution misalignments is covariant under rotations, a Kochen-Specker theorem for the unsharp spin observables follows: There are finite sets of directions such that not all the unsharp spin observables in these directions can consistently be assigned approximate truth-values in a non-contextual way.
\end{abstract}

\section{Introduction}

The Kochen-Specker (KS) theorem establishes that not all measurement outcomes predicted by quantum mechanics can result from detecting hypothetically predetermined values of the observables. Recently, there have been debates whether or not this result is relevant for finite-precision measurements [14, 12, 9, 5, , $1,3,15$, 16, 11, 6, 1. The goal of this paper is (1) to give a sound description of measurements with a residual uncertainty about the actual orientation of the measurement device, (2) to establish a KS-type no-hidden-variables theorems for this kind of finite precision measurements. For the sake of definiteness I will discuss these issues for a spin 1 particle, but similar results hold for arbitrary observables in a Hilbert space of dimension 3 or higher.

In Section 2 I will construct POV-observables representing finite precision measurements of a spin 1 particle. I will also discuss some important properties of these unsharp spin observables. In Section 3 I will prove a KS-theorem for these

\footnotetext{
${ }^{1}$ Work partially supported by the Austrian Fonds zur Förderderung der wissenschaftlichen Forschung, Spezialforschungsbereich SFB F012 at the Universität Salzburg.
} 
unsharp spin observables. The paper concludes with a discussion of the relation of these results to the current debate on the validity of KS-theorems for finite precision measurements.

\section{$2 \quad$ Finite Precision Measurements of Spin 1-Observables}

The sharp spin 1 observables in $\boldsymbol{x}, \boldsymbol{y}$, and $\boldsymbol{z}$ direction are given by the three threedimensional Pauli matrices $S_{\boldsymbol{x}}, S_{\boldsymbol{y}}, S_{\boldsymbol{z}}$, each of which has eigenvalues 1,0 , and -1 . For example $S_{\boldsymbol{z}}$ is given by

$$
S_{z}=\left(\begin{array}{ccc}
1 & 0 & 0 \\
0 & 0 & 0 \\
0 & 0 & -1
\end{array}\right) .
$$

Denote the eigenvectors of the spin matrix $S_{\boldsymbol{z}}$ by $\psi_{\boldsymbol{z}, 1}, \psi_{\boldsymbol{z}, 0}, \psi_{\boldsymbol{z},-1}$ and the corresponding eigenprojectors by $P_{\boldsymbol{z}, 1}:=\left|\psi_{\boldsymbol{z}, 1}\right\rangle\left\langle\psi_{\boldsymbol{z}, 1}\right|$ and similarly for $P_{\boldsymbol{z}, 0}$ and $P_{\boldsymbol{z},-1}$. The $P_{\boldsymbol{z}, i}$ are sharp spin properties. For example,

$$
P_{\boldsymbol{z}, 1}=\left(\begin{array}{ccc}
1 & 0 & 0 \\
0 & 0 & 0 \\
0 & 0 & 0
\end{array}\right) .
$$

Similar notation will be used for the $\boldsymbol{x}$ - and the $\boldsymbol{y}$-axes.

For an arbitrary direction $\boldsymbol{n}$ the sharp spin 1 observable is $S_{\boldsymbol{n}}:=\boldsymbol{n} \cdot \boldsymbol{S}$, where $\boldsymbol{S}$ is the Pauli vector $\left(S_{\boldsymbol{x}}, S_{\boldsymbol{y}}, S_{\boldsymbol{z}}\right) . S_{\boldsymbol{n}}$ also has eigenvalues 1,0 , and -1 . Let $\psi_{\boldsymbol{n}, i}$ and $P_{n, i}$ be the eigenstates and eigenprojectors of $S_{\boldsymbol{n}}$ corresponding to the eigenvalues $i=1,0,-1$. The sharp spin observable $S_{\boldsymbol{n}}$ in direction $\boldsymbol{n}$ can be represented as a projection valued (PV)-measure on the value space $\Omega=\{1,0,-1\}$, which associates to each element $i$ of the value space $\Omega$ the projector $P_{\boldsymbol{n}, i}$.

Now assume we are not sure that we actually measure the spin in the direction $\boldsymbol{n}$ we want. We only know that the directions $\boldsymbol{m}$ of actual spin measurements is distributed with a density $w_{\boldsymbol{n}, \epsilon}(\boldsymbol{m})$ around the intended direction $\boldsymbol{n}$. The probability that such an imprecisely specified measurement yields an outcome +1 when the spin 1 system is prepared in some pure state $\psi$ is $\operatorname{Prob}_{\psi}^{\boldsymbol{n}, \epsilon}(+1)=$ $\int_{S^{2}} d \Omega(\boldsymbol{m}) w_{\boldsymbol{n}, \epsilon}(\boldsymbol{m}) \operatorname{tr}\left(P_{\psi} P_{\boldsymbol{m},+1}\right)=\operatorname{tr}\left(P_{\psi} \int_{S^{2}} d \Omega(\boldsymbol{m}) w_{\boldsymbol{n}, \epsilon}(\boldsymbol{m}) P_{\boldsymbol{m},+1}\right)$, where $d \Omega$ is the Lesbesgue-measure of the sphere. Defining

$$
F^{\boldsymbol{n}, \epsilon}(i):=\int_{S^{2}} w_{\boldsymbol{n}, \epsilon}(\boldsymbol{m}) P_{\boldsymbol{m}, i} d \Omega(\boldsymbol{m}) .
$$

the probability of outcome $i$ can be written as

$$
\operatorname{Prob}_{\psi}^{\boldsymbol{n}, \epsilon}(i)=\operatorname{tr}\left(P_{\psi} F^{\boldsymbol{n}, \epsilon}(i)\right) .
$$

From (3) it is obvious that the $F^{\boldsymbol{n}, \boldsymbol{\epsilon}}$ are positive self-adjoint operators satisfying $0 \leq F^{\boldsymbol{n}, \epsilon} \leq \mathbb{I}$. But they are not projectors since $F^{\boldsymbol{n}, \epsilon}(i) \neq F^{\boldsymbol{n}, \epsilon}(i)^{2}$. The $F^{\boldsymbol{n}, \epsilon}(i)$ form a resolution of the identity,

$$
F^{\boldsymbol{n}, \epsilon}(1)+F^{\boldsymbol{n}, \epsilon}(0)+F^{\boldsymbol{n}, \epsilon}(-1)=\mathbb{I},
$$


which follows from (3) and $P_{\boldsymbol{n}, 1}+P_{\boldsymbol{n}, 0}+P_{\boldsymbol{n},-1}=\mathbb{1}$. Thus we have a positive operator valued measure (POVM) $F^{\boldsymbol{n}, \boldsymbol{\epsilon}}$ which associates to each element $i$ of the value space $\Omega$ the projector $F^{\boldsymbol{n}, \epsilon}(i)$. Because of (4i) this measure is normalised. POV-measures are the standard tool for describing realistic experiments [2, 跑.

Proposition 1 If the distributions $w_{\boldsymbol{n}, \epsilon}$ of apparatus misalignments transform co-

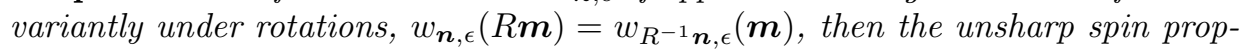
erties $F^{\boldsymbol{n}, \epsilon}$ transform covariantly under rotations,

$$
D^{1}(R) F^{\boldsymbol{n}, \epsilon}(i) D^{1}(R)^{-1}=F^{R^{-1} \boldsymbol{n}, \epsilon}(i),
$$

where $D^{1}$ is the spin 1-representation of the rotation group.

To see this note that the sharp spin properties $P_{m i}$ are angular momentum operators and therefore transform covariantly [2, p. 70],

$$
D^{1}(R) P_{\boldsymbol{m}, i} D^{1}(R)^{-1}=P_{R^{-1} \boldsymbol{m}, i} .
$$

Using this and and the definition of the unsharp spin properties (3), we obtain $D^{1}(R) F^{\boldsymbol{n}, \epsilon}(i) D^{1}(R)^{-1}=\int_{S^{2}} w_{\boldsymbol{n}, \epsilon}(\boldsymbol{m}) P_{R^{-1} \boldsymbol{m}, i} d \Omega(\boldsymbol{m})$. Introducing new coordinates $\boldsymbol{m}^{\prime}=R^{-1} \boldsymbol{m}$, and using the rotation invariance of $d \Omega$ and $w_{\boldsymbol{n}, \epsilon}(R \boldsymbol{m})=$ $w_{R^{-1} \boldsymbol{n}, \epsilon}(\boldsymbol{m})$, the right hand side is seen to equal $F^{R^{-1} \boldsymbol{n}, \epsilon}(i)$, which establishes (河).

Since the $F^{\boldsymbol{n}, \epsilon}(i)$ transform covariantly under rotations, they are angular momentum properties and can be regarded as spin properties with the same justification as the sharp spin properties $P_{\boldsymbol{n}, i}$. This is in line with Weyl's idea of defining observables by their transformation properties under some kinematic group.

Proposition 2 If the distribution $w_{\boldsymbol{n}, \epsilon}$ of measurement errors transforms covariantly under rotations, $w_{\boldsymbol{n}, \epsilon}(R \boldsymbol{m})=w_{R^{-1} \boldsymbol{n}, \epsilon}(\boldsymbol{m})$, then every eigenvector of the sharp spin properties $P_{\boldsymbol{m} i}$ is also an eigenvector of the unsharp spin properties $F^{\boldsymbol{n}, \epsilon}(i)$. Since the sharp spin properties $\left\{P_{\boldsymbol{n}, i}\right\}_{i=1,0,-1}$ have simultaneous eigenvectors and commute, this is also the case for the unsharp spin properities $\left\{F^{\boldsymbol{n}, \epsilon}(i)\right\}_{i=1,0,-1}$.

To see this, we proceed in two steps. In a first step we consider the special case that the intended measurement direction $\boldsymbol{n}$ is the $\boldsymbol{z}$-direction. The eigenvectors of $P_{\boldsymbol{z}, i}$ are the vectors in the $\boldsymbol{x}, \boldsymbol{y}$, or $\boldsymbol{z}$-directions. We will show that $F^{\boldsymbol{n}, \boldsymbol{\epsilon}}$ has the same eigenvectors.

Using the explicit form of the spin matrices $S_{\boldsymbol{x}}, S_{\boldsymbol{y}}, S_{\boldsymbol{z}}$, one can calculate the $P_{\boldsymbol{m}, i}$. For $i=1$ and $\boldsymbol{m}$ in polar coordinates $(\theta, \phi)$ it turns out that $P_{\boldsymbol{m}(\theta, \phi), 1}$ equals

$$
\left(\begin{array}{ccc}
\cos \left(\frac{\theta}{2}\right)^{4}, & \frac{1}{2 \sqrt{2}} e^{-i \phi}(1+\cos (\theta)) \sin (\theta) & \frac{1}{4} e^{2 i \phi} \sin (\theta)^{2} \\
\frac{1}{2 \sqrt{2}} e^{i \phi}(1+\cos (\theta)) \sin (\theta) & \frac{1}{2} \sin (\theta)^{2} & \sqrt{2} e^{-i \phi} \cos \left(\frac{\theta}{2}\right) \sin \left(\frac{\theta}{2}\right)^{3} \\
\frac{1}{4} e^{-2 i \phi} \sin (\theta)^{2} & \sqrt{2} e^{i \phi} \cos \left(\frac{\theta}{2}\right) \sin \left(\frac{\theta}{2}\right)^{3} & \sin \left(\frac{\theta}{2}\right)^{4}
\end{array}\right)
$$


The covariance property of $w_{\boldsymbol{n}, \epsilon}$ implies that $w_{\boldsymbol{n}, \epsilon}$ is invariant under rotations around $\boldsymbol{n}$ : If $R$ is a rotation around $\boldsymbol{n}$, then $w_{\boldsymbol{n}, \epsilon}(R \boldsymbol{m})=w_{R^{-1} \boldsymbol{n}, \epsilon}(\boldsymbol{m})=$ $w_{\boldsymbol{n}, \epsilon}(\boldsymbol{m})$. Since $w_{\boldsymbol{z}, \epsilon}$ is invariant under all rotations around the $\boldsymbol{z}$-axis, we can write $w_{\boldsymbol{z}, \epsilon}(\boldsymbol{m})=w_{\boldsymbol{z}, \epsilon}(\boldsymbol{m}(\theta, \phi))=: w_{\boldsymbol{z}, \epsilon}(\theta)$, where $(\theta, \phi)$ are the polar coordinates of the unit vector in the direction $\boldsymbol{m}$. Thus we have

$$
\int_{0}^{2 \pi} w_{\boldsymbol{z}, \epsilon}(\theta, \phi) P_{\boldsymbol{m}(\theta, \phi), 1} d \phi=w_{\boldsymbol{z}, \epsilon}(\theta) \int_{0}^{2 \pi} P_{\boldsymbol{m}(\theta, \phi), 1} d \phi .
$$

Evaluating the integral on the right hand side yields the matrix

$$
\left(\begin{array}{ccc}
2 \pi \cos \left(\frac{\theta}{2}\right)^{4}, & 0 & 0 \\
0 & \pi \sin (\theta)^{2} & 0 \\
0 & 0 & 2 \pi \sin \left(\frac{\theta}{2}\right)^{4}
\end{array}\right) .
$$

Therefore

$$
\begin{aligned}
F^{\boldsymbol{z}, \epsilon}(1) & =\int_{S^{2}} w_{\boldsymbol{z}, \epsilon}(\boldsymbol{m}) P_{\boldsymbol{m}, i} d \Omega(m) \\
& =\int_{0}^{\pi} d \theta \sin (\theta) \int_{0}^{2 \pi} d \phi w_{\boldsymbol{z}, \epsilon}(\theta, \phi) P_{\boldsymbol{m}(\theta, \phi), 1} \\
& =\left(\begin{array}{ccc}
\alpha_{1} & 0 & 0 \\
0 & \alpha_{2} & 0 \\
0 & 0 & \alpha_{3}
\end{array}\right) .
\end{aligned}
$$

Similarly one can derive

$$
F^{\boldsymbol{z}, \epsilon}(0)=\left(\begin{array}{ccc}
\alpha_{2} & 0 & 0 \\
0 & \alpha_{4} & 0 \\
0 & 0 & \alpha_{2}
\end{array}\right) \quad F^{\boldsymbol{z}, \epsilon}(-1)=\left(\begin{array}{ccc}
\alpha_{3} & 0 & 0 \\
0 & \alpha_{2} & 0 \\
0 & 0 & \alpha_{1}
\end{array}\right),
$$

where

$$
\begin{aligned}
& \alpha_{1}=2 \pi \int_{0}^{\pi} d \theta w_{\boldsymbol{z}, \epsilon}(\theta) \sin (\theta) \cos (\theta / 2)^{4} \\
& \alpha_{2}=\pi \int_{0}^{\pi} d \theta w_{\boldsymbol{z}, \epsilon}(\theta) \sin (\theta) \sin (\theta)^{2} \\
& \alpha_{3}=2 \pi \int_{0}^{\pi} d \theta w_{\boldsymbol{z}, \epsilon}(\theta) \sin (\theta) \sin (\theta / 2)^{4} . \\
& \alpha_{4}=2 \pi \int_{0}^{\pi} d \theta w_{\boldsymbol{z}, \epsilon}(\theta) \sin (\theta) \cos (\theta)^{2}
\end{aligned}
$$

The $F^{\boldsymbol{z}, \epsilon}(i)$ are diagonal matrices. Therefore the unit vectors in the $\boldsymbol{x}, \boldsymbol{y}$, and $\boldsymbol{z}$-directions are eigenvectors of all $F^{\boldsymbol{z}, \epsilon}(i)$. This finishes the proof of Proposition 2 for the special case $\boldsymbol{n}=\boldsymbol{z}$.

In the second step we consider the general case that we want to measure spin in an arbitrary direction $\boldsymbol{n}$. First we will show that the eigenvectors of $\boldsymbol{F}^{\boldsymbol{n}, \epsilon}(i)$ are $D^{1}(R)^{-1} \boldsymbol{x}, D^{1}(R)^{-1} \boldsymbol{y}, D^{1}(R)^{-1} \boldsymbol{z}$, where $R$ is a rotation fulfilling $R^{-1} \boldsymbol{n}=$ 
$\boldsymbol{z}$. Using the covariance property (5) and the fact that $\boldsymbol{x}, \boldsymbol{y}, \boldsymbol{z}$ are eigenvectors of the $F^{\boldsymbol{z}, \epsilon}(i)$ we verify $F^{\boldsymbol{n}, \epsilon}(i) D^{1}(R)^{-1} \boldsymbol{x}=D^{1}(R)^{-1} F^{\boldsymbol{z}, \epsilon}(i) D^{1}(R) D^{1}(R)^{-1} \boldsymbol{x}=$ $D^{1}(R)^{-1} F^{\boldsymbol{z}, \epsilon}(i) \boldsymbol{x}=D^{1}(R)^{-1} \alpha \boldsymbol{x}=\alpha D^{1}(R)^{-1} \boldsymbol{x}$, which implies that $D^{1}(R)^{-1} \boldsymbol{x}$ is an eigenvector of $F^{\boldsymbol{n}, \epsilon}(i)$. Similarly we can check that $D^{1}(R)^{-1} \boldsymbol{y}$ and $D^{1}(R)^{-1} \boldsymbol{z}$ are eigenvectors of $F^{\boldsymbol{n}, \epsilon}(i)$. Since $F^{\boldsymbol{n}, \epsilon}(i)$ only has three eigenvectors, $D^{1}(R)^{-1} \boldsymbol{x}$, $D^{1}(R)^{-1} \boldsymbol{y}, D^{1}(R)^{-1} \boldsymbol{z}$ are the only eigenvectors of $F^{\boldsymbol{n}, \epsilon}(i)$.

Next we will show that $D^{1}(R)^{-1} \boldsymbol{x}, D^{1}(R)^{-1} \boldsymbol{y}, D^{1}(R)^{-1} \boldsymbol{z}$ are the eigenvectors of $P_{n, i}$. Using the covariance property (6) of the sharp spin observables and the fact that $\boldsymbol{x}, \boldsymbol{y}, \boldsymbol{z}$ are eigenvectors of the $P_{\boldsymbol{z}, i}$ with eigenvalues $\lambda=1,0,-1$ we verify $P_{\boldsymbol{n}, i} D^{1}(R)^{-1} \boldsymbol{x}=D^{1}(R)^{-1} P_{\boldsymbol{n}, i} D^{1}(R) D^{1}(R)^{-1} \boldsymbol{x}=D^{1}(R)^{-1} P_{\boldsymbol{n}, i} \boldsymbol{x}=$ $D^{1}(R)^{-1} \lambda \boldsymbol{x}=\lambda D^{1}(R)^{-1} \boldsymbol{x}$, which implies that $D^{1}(R)^{-1} \boldsymbol{x}$ is an eigenvector of $P_{\boldsymbol{n}, i}$. Similarly we can check that $D^{1}(R)^{-1} \boldsymbol{y}$ and $D^{1}(R)^{-1} \boldsymbol{z}$ are eigenvectors of $P_{\boldsymbol{n}, i}$. Since $P_{\boldsymbol{n}, i}$ only has three eigenvectors, $D^{1}(R)^{-1} \boldsymbol{x}, D^{1}(R)^{-1} \boldsymbol{y}, D^{1}(R)^{-1} \boldsymbol{z}$ are the only eigenvectors of $P_{\boldsymbol{n}, i}$. Thus the $P_{\boldsymbol{n}, i}$ and the $F^{\boldsymbol{n}, \epsilon}(i)$ all have the same three eigenvectors. This establishes Proposition 2 for the general case.

Proposition 3 The eigenvalues of the unsharp spin properties $F^{\boldsymbol{n}, \epsilon}(i)$ are in the set $\left\{\alpha_{1}, \ldots, \alpha_{4}\right\}$, where the $\alpha_{i}$ are given by equations (9). The eigenvalues of each $F^{\boldsymbol{n}, \epsilon}(i)$ add up to 1.

By the covariance property (5) of the $F^{\boldsymbol{n}, \epsilon}(i)$, the $F^{\boldsymbol{n}, \epsilon}(i)$ have the same eigenvalues as $F^{\boldsymbol{z}, \epsilon}(i)$. The eigenvalues of the $F^{\boldsymbol{z}, \epsilon}(i)$ are the $\alpha_{i}$ of equations (9). Taking into account that $1=\int_{S^{2}} d \Omega(\boldsymbol{m}) w_{\boldsymbol{n}, \epsilon}(\boldsymbol{m})=2 \pi \int_{0}^{\pi} d \theta \sin (\theta) w_{\boldsymbol{n}, \epsilon}(\theta)$ one easily verifies from (9) that $0 \leq \alpha_{i} \leq 1$ and $\alpha_{1}+\alpha_{2}+\alpha_{3}=1, \alpha_{2}+\alpha_{4}+\alpha_{2}=1$.

To give an explicit example, assume that the spin directions actually measured are uniformly distributed over $C(\boldsymbol{n}, \epsilon)$, the set of directions deviating from $\boldsymbol{n}$ by less than an angle $\epsilon$. Denote by $A$ the size of $C(\boldsymbol{n}, \epsilon)$ on the unit sphere. So $w_{\boldsymbol{n}, \epsilon}$ is $1 / A$ times the characteristic function of $C(\boldsymbol{n}, \epsilon)$. The $F^{\boldsymbol{z}, \epsilon(i)}$ have the form (7) and (8) with

$$
\begin{aligned}
\alpha_{1} & =\frac{1}{24}(15+8 \cos (\epsilon)+\cos (2 \epsilon)) \\
\alpha_{2} & =\frac{1}{3}(2+\cos (\epsilon)) \sin (\epsilon / 2)^{2} \\
\alpha_{3} & =\frac{1}{3} \sin (\epsilon / 2)^{4} \\
\alpha_{4} & =\frac{1}{6}(3+2 \cos (\epsilon)+\cos (2 \epsilon)) .
\end{aligned}
$$

Observe that, as the measurement inaccuracy $\epsilon$ tends to zero, two eigenvalues ( $\alpha_{2}$ resp. $\left.\alpha_{3}\right)$ of each $F^{\boldsymbol{z}, \epsilon}(i)$ go to zero, one eigenvalue $\left(\alpha_{1}\right.$ resp. $\left.\alpha_{4}\right)$ goes to one. Comparing (77) to (2) we see that the unsharp spin properties $F^{\boldsymbol{z}, \epsilon}(i)$ converge to the sharp spin properties $P_{\boldsymbol{z}, i}$ as the measurement inaccuracy goes to zero.

Additionally, given any vector which is an eigenvector of the $F^{\boldsymbol{n}, \epsilon}(i)$, for one of the $F^{\boldsymbol{n}, \epsilon}(i)$ this vector has an eigenvalue close to one, and for the two other $F^{\boldsymbol{n}, \epsilon}(i)$, this vector has an eigenvalue close to zero. For example, the vector $(0,1,0)$ 
is an eigenvector of $F^{\boldsymbol{z}, \epsilon}(0)$ with eigenvalue $\alpha_{4}$ (which is close to one), and it is an eigenvector of $F^{\boldsymbol{z}, \epsilon}(1)$ and $F^{\boldsymbol{z}, \epsilon}(-1)$ with eigenvalue $\alpha_{2}$ (which is close to zero).

\section{The KS-theorem for Unsharp Spin 1 Observ- ables}

Determining the result $i \in\{1,0,-1\}$ of a sharp spin measurement in direction $\boldsymbol{n}$ is picking one of the sharp spin properties $\left\{P_{\boldsymbol{n}, i}\right\}_{i=1,0,-1}$ and assigning it the truth value 1. Since the sharp spin properties are projectors $P_{\boldsymbol{n}, i}:=\left|\psi_{\boldsymbol{n}, i}\right\rangle\left\langle\psi_{\boldsymbol{n}, i}\right|$ they can be identified with the rays $\psi_{\boldsymbol{n}, i}$. So, assigning the value 1 to one of the $P_{\boldsymbol{n}, i}$ and the value 0 to the other two, is equivalent to assigning the colour $\mathrm{T}$ (true) to one of the rays $\psi_{\boldsymbol{n}, i}$ and the colour $\mathrm{F}$ (false) to the two other rays. The traditional KS-proofs show that for certain sets of directions this colouring rule cannot be satisfied.

Determining the result $i \in\{1,0,-1\}$ of an unsharp spin measurement in direction $\boldsymbol{n}$ is picking one of the unsharp spin properties $\left\{F^{\boldsymbol{n}, \epsilon}(i)\right\}_{i=1,0,-1}$ and assigning it the truth value 1 . But the unsharp spin properties are not projectors and therefore cannot readily be identified with rays. To arrive at a colouring rule for rays we have to proceed in a different way.

Assume for example that one intends to measure spin in direction $\boldsymbol{z}$ and result 0 occurs. This means that the unsharp spin property $F^{\boldsymbol{z}, \epsilon}(0)$ is realised, whereas $F^{\boldsymbol{z}, \epsilon}(1)$ and $F^{\boldsymbol{z}, \epsilon}(-1)$ are not realised. Accordingly we assign the colour AT (almost true) to the ray $(0,1,0)$, which is the eigenvector of $F^{\boldsymbol{z}, \epsilon}(0)$ with eigenvalue close to 1 . To $(1,0,0)$ and $(0,0,1)$ we assign the colour AF (almost false) because they are eigenvectors of $F^{\boldsymbol{z}, \epsilon}(0)$ with eigenvalue close to 0 . Had the outcome been 1 , we would have assigned AT to $(1,0,0)$ and AF to $(0,1,0)$ and to $(0,0,1)$. This is achieved by the following value assignment.

Definition 1 Fix some unsharpness tolerance $0.5>\delta \geq 0$. If the outcome of a spin measurement with some intended direction $\boldsymbol{n}$ is some $i \in\{1,0,-1\}$, then the ray of the eigenvector of $F^{\boldsymbol{n}, \epsilon}(i)$ corresponding to an eigenvalue larger or equal to $1-\delta$ gets colour $A T$, and the rays of eigenvectors corresponding to eigenvalues smaller or equal to $\delta$ get colour AF. Rays corresponding to some eigenvalue between $\delta$ and $1-\delta$ are assigned a third colour or no colour.

$\delta>0$ is an unsharpness tolerance below which an eigenvalue counts as "almost zero". An eigenvalue above $1-\delta$ counts as "almost one". The exact level is a matter of taste, and our results do not depend on the exact level. But certainly $\delta$ should be smaller than 0.5 , since otherwise some values would simultaneously be counted as almost zero and almost one.

In a way this assignment of approximate truth values is conservative: a state assigns approximate truth values only to those unsharp spin properties of which it is an eigenstate. Note that if $F^{\boldsymbol{n}, \epsilon}(i)$ possesses an approximate truth value, it does possess it without dispersion. Contrast this with a situation where the spin 
in some direction $\boldsymbol{n}^{\prime}$ close to $\boldsymbol{n}$ has value $i$; the spin value in direction $\boldsymbol{n}$ is not dispersion-free.

Note that the intended measurement direction $\boldsymbol{n}$ need not be among the coloured rays. An example: If $\boldsymbol{n}=\boldsymbol{x}$, the rays which are coloured are the normalised eigenvectors of the $F^{\boldsymbol{x}, \epsilon}(i)$. By Proposition 2 these are exactly the rays of the eigenvectors of $P_{\boldsymbol{x}, i}$. The eigenvectors of the $P_{\boldsymbol{x}, i}$ are by definition the eigenvectors of $S_{\boldsymbol{x}}$, namely $(1,-\sqrt{2}, 1) / 2,(-1,0,1) / \sqrt{2}$, and $(1, \sqrt{2}, 1) / 2$. The direction $\boldsymbol{x}$ is not coloured. Note also that we are colouring complex rays, not real rays.

In Proposition 2 we have seen that for a fixed intended measurement direction $\boldsymbol{n}$ the $\left\{F^{\boldsymbol{n}, \epsilon}(i)\right\}_{i=1,0,-1}$ have the same eigenvectors. If the measurement inaccuracy is sufficiently small - or to me more precise: if the density $w_{\boldsymbol{n}, \epsilon}(\boldsymbol{m})$ of apparatus misalignments has enough probability mass sufficiently close to the intended measurement direction $\boldsymbol{n}$ - then the eigenvalues $\alpha_{1}$ and $\alpha_{4}$ of equations (9) will be larger than $1-\delta$, whereas $\alpha_{2}$ and $\alpha_{3}$ will be smaller than $\delta$. If this is the case, for each direction $\boldsymbol{n}$, exactly one ray in the orthogonal triad of eigenvectors of the $F^{\boldsymbol{n}, \epsilon}(i)$ will get colour AT, and two rays will get colour AF.

For example if we assume apparatus misalignments to be uniformly distributed over the set of directions deviating by less than an angle $\epsilon$ and choose $\delta=0.1$, then we can calculate from equations $(10)$ that for $\epsilon$ smaller than $0.459=26.3^{\circ}$ condition (2) is fulfilled and $\alpha_{1}, \alpha_{4}$ will be larger than $0.9, \alpha_{2}, \alpha_{3}$ will be smaller than 0.1. So for $\delta=0.1$, if the measurement inaccuracy $\epsilon$ is smaller than 0.459 , then in all orthogonal tripods one ray will be coloured AT and two rays will be coloured AF.

Let $N$ denote a set of directions, and $F(N, \epsilon)$ the set of positive operators in the ranges of the $F^{\boldsymbol{n}, \epsilon}$ with $n \in N$. Let $V(N, \epsilon)$ the set of rays of the eigenvectors of the $F^{\boldsymbol{n}, \epsilon}$ with $n \in N$. Then, a hidden variable model can uniquely determine the measured values of spin measurements in all directions of $N$ only if there exists a colouring $V(N, \epsilon) \rightarrow\{A T, A F\}$ which associates for all directions $\boldsymbol{n} \in N$ the colour AT to one of the eigenvectors of the $F^{\boldsymbol{n}, \epsilon}(i)$ and the colour AF to the two other eigenvectors.

One ray can be eigenvector of spin properties $F^{\boldsymbol{n}, \epsilon}(i), F^{\boldsymbol{m}, \epsilon}(i)$ in different directions $\boldsymbol{n}, \boldsymbol{m}$. Non-contextuality of the hidden variable-model implies that such a ray is assigned a unique colour. Now the KS-theorem for unsharp spin observables follows in exactly the same way as the one for sharp observables. In every orthogonal tripod one of the rays is constrained to get the colour AT, the other two rays get AF. For the KS-sets of tripods such a colouring is impossible [10, 13, 17]. Thus we arrive at the following

Theorem 1 For any unsharpness tolerance $0.5>\delta \geq 0$, if in an unsharp spin 1 measurement

(1) the densities of apparatus misalignments transform covariantly, $w_{\boldsymbol{n}, \epsilon}(R \boldsymbol{m})=$ $w_{R^{-1} \boldsymbol{n}, \epsilon}(\boldsymbol{m})$, and

(2) the measurement inaccuracy described by the densities $w_{\boldsymbol{n}, \epsilon}(\boldsymbol{m})$ of apparatus misalignments is so small that in equations (9) $\alpha_{1}$ and $\alpha_{4}$ are larger or equal to $1-\delta$, while $\alpha_{2}$ and $\alpha_{3}$ are smaller or equal to $\delta$, 
then not all the unsharp spin observables in a KS-set of directions can consistently be assigned approximate truth-values in a non-contextual way.

\section{Discussion}

Let me briefly relate this result to the debate on the relevance of the KS-theorem for inaccurate measurements. This debate was fueled by claims of Meyer [12], Kent [9], and Clifton and Kent [5], MKC for short, that for finite precision measurements the KS-theorem is irrelevant.

Infinite precision is crucial to the KS-argument in two ways [7]: (1) It is necessary to the argument that the measured components of one tripod are exactly orthogonal. (2) In order to exploit non-contextuality it is necessary that two measurements intended to pick out the same observable as member of two different maximal sets pick out exactly the same direction. MKC show that non-contextual hidden variable models can be constructed if we relax the assumption of infinite precision and allow for an arbitrarily small violation of (2). In these models it is not exactly the directions in a KS-set that are assigned non-contextual values, but points which approximate them arbitrarily closely. In fact it is possible to assign values to a dense set of observables. (In our spin 1 case this amounts to a colouring of a dense subset of the unit sphere.)

So the hidden variable-theorist is free to adopt the hypothesis that due to some apparatus misalignment instead of the intended observable he measures another observable, which cannot be distinguished from the intended observable by a finite precision measurement. The results of these finite precision measurements can be explained by a non-contextual hidden variable model.

In the MKC model the distribution $w_{\boldsymbol{n}, \epsilon}(\boldsymbol{m})$ of misalignments is not rotation covariant: Assume that instead of an intended direction $\boldsymbol{n}$ the apparatus is in fact aligned in a direction $\boldsymbol{m}$ with rational coordinates, which is very close to $\boldsymbol{n}$. Now let $R$ be a rotation by $45^{\circ}$ around an axis orthogonal to $\boldsymbol{m}$. If $w_{\boldsymbol{n}, \epsilon}(\boldsymbol{m})$ were rotation covariant, in an experiment designed to measure spin in direction $R \boldsymbol{n}$ we would actually measure spin in direction $R \boldsymbol{m}$. So the result of a measurement in intended direction $R \boldsymbol{n}$ would be determined by the colour assigned to the point $R \boldsymbol{m}$. But $R \boldsymbol{m}$ cannot have rational coordinates if $\boldsymbol{m}$ has. So $R \boldsymbol{m}$ would not be assigned a colour in Meyer's model and the result of a measurement in intended direction $R \boldsymbol{n}$ would not be predetermined. The result of a measurement in intended direction $R \boldsymbol{n}$ must therefore be determined by the colour of some point other than $R \boldsymbol{m}$. Thus the distribution $w_{\boldsymbol{n}, \epsilon}(\boldsymbol{m})$ of misalignments in the MKC-models cannot not be covariant under all rotations. In Meyer's model condition (1) of Theorem 1 is not satisfied.

From the more operational point of view taken in this paper, the observer who wants to measure spin in a direction $\boldsymbol{n}$ will have an experimental procedure for trying to do this as exactly as possible. Simon et al. [16] refer to this procedure by saying he sets the "control switch" of his apparatus to the position $\boldsymbol{n}$. The switch position is all the observer knows about. In an operational sense, the physical 
observable measured is entirely determined by the switch position. However, there will usually be degrees of freedom of the apparatus which the experimenter cannot control. This results in an apparatus misalignment of which the experimenter is not aware. If he were aware of it he would correct it. Not being aware of the misalignment he interprets the outcome produced by the misaligned apparatus as result of an experiment without misalignment.

Unlike Simon et al [16] I do not describe the misalignment by associating hidden variables to the apparatus. Rather I describe the effects of the misalignment by the unsharp spin observables $F^{\boldsymbol{n}, \epsilon}$. These are constructed so as to yield exactly the statistics of outcomes associated to the switch positions. Theorem 1 i shows that the results of measurements with misalignments distributed in a rotation covariant way cannot be predetermined by a non-contextual hidden variable model.

\section{References}

[1] Appleby D. M. (2001), "Nullification of the Nullification", quant-ph/0109034

[2] Busch P., Grabowski M., Lahti P. (1995), Operational Quantum Physics, LNP m31, Heidelberg: Springer

[3] Cabello A. (2001). "Finite Precision Measurement does not Nullify the Kochen-Specker Theorem", quant-ph/0104024

[4] Cabello A., García-Alcaine G. (1998), "Proposed_Experimental Test of the Bell-KochenSpecker Theorem", Phys. Rev. Lett. 80, 1797. quant-ph/9709047

[5] Clifton R., Kent A. (2000), "Simulating Quantum_Mechanics by Non-contextual Hidden Variables", Proc. R. Soc. Lond. A 456, 2101. quant-ph/9908031

[6] Havlicek H., Krenn G., Summhammer J., Svozil K. (2001), "Coloring the Rational Quantum Sphere and the Kochen-Specker Theorem", J. Phys. A 343, 3071. quant-ph/9911040

[7] Held C., The Kochen-Specker Theorem. Stanford Encyclopedia of Philosophy. Accessible at hppt://plate.stanford.edu/entries/kochen-specker/index.html

[8] Holevo A. S. (1982), Probabilistic and Statistical Aspects of Quantum Theory, Amsterdam: North Holland

[9] Kent A. (1999), "Non-Contextual Hidden Variables and Physical Measurements", Phys. Rev. Lett. 83, 3755. Also quant-ph/9906006

[10] Kochen S., Specker E. (1967), "The Problem of Hidden Variables in Quantum Mechanics", J. Math. Mech 17, 59-87

[11] Mermin D. (1999), "A Kochen-specker Theorem for Imprecisely Specified Measurements", quant-ph/9912081

[12] Meyer D. A. (1999), "Finite Precision Measurement Nullifies the Kochen-Specker Theorem", Phys. Rev. Lett. 83, 3751. quant-ph/9905080

[13] Peres A. (1995), Quantum Thory: Concepts and Methods, Dordrecht: Kluwer

[14] Pitovsky I. (1985), Quantum Mechanics and Value Definiteness, Phil. Sci. 52, 154-156

[15] Simon C., Zukowski M., Weinfurter H., Zeilinger A. (2000), "A Feasible Kochen-Specker Experiment with Single Particles", Phys. Rev. Lett. 85, 1783. quant-ph/0009074

[16] Simon C., Bruckner C., Zeilinger A. (2001). "Hidden Variable Theorems for Real Experiments", Phys. Rev. Lett 86, 4427. quant-ph/0006043

[17] Zimba J., Penrose R. (1993), "On Bell Non-Locality without Probabilities: More Curios Geometry", Stud. Hist. Phil. Sci. 24, 697 